\title{
Improving the Interpretability of Random Forest Models of Genetic Association in the Presence of Non-Additive Interactions
}

\author{
Alena Orlenko ( $\square$ aorlenko@upenn.edu ) \\ University of Pennsylvania https://orcid.org/0000-0003-1757-293X \\ Jason H Moore \\ University of Pennsylvania
}

Research article

Keywords: Machine learning, feature importances, random forest, epistasis, simulation, Alzheimer's disease, glaucoma

Posted Date: May 21st, 2020

DOI: https://doi.org/10.21203/rs.3.rs-29752/v1

License: (c) (i) This work is licensed under a Creative Commons Attribution 4.0 International License. Read Full License 


\section{Abstract}

Background Non-additive interactions among genes are frequently associated with a number of phenotypes, including known complex diseases such as Alzheimer's, diabetes, and cardiovascular disease. Detecting interactions requires careful selection of analytical methods, and some machine learning algorithms are unable or underpowered to detect or model feature interactions that exhibit non-additivity. The Random Forest method is often employed in these efforts due to its ability to detect and model non-additive interactions. In addition, Random Forest has the built-in ability to estimate feature importance scores, a characteristic that allows the model to be interpreted with the order and effect size of the feature association with the outcome. This characteristic is very important for epidemiological and clinical studies where results of predictive modeling could be used to define the future direction of the research efforts. An alternative way to interpret the model is with a permutation feature importance metric which employs a permutation approach to calculate a feature contribution coefficient in units of the decrease in the model's performance. Currently, it is unclear which Random Forest feature importance metric provides a superior estimation of the true informative contribution of features in genetic association analysis.

Results To address this issue, and to improve interpretability of Random Forest predictions, we compared different methods for feature importance estimation in real and simulated datasets with non-additive interactions. As a result, we detected a discrepancy between the metrics evaluations for the real-world datasets and further established that the permutation feature importance metric provides more precise feature importance rank estimation for the simulated datasets with non-additive interactions.

Conclusions By analyzing both real and simulated data, we established that the permutation feature importance metric provides more precise feature importance rank estimation in the presence of non-additive interactions.

\section{Background}

Machine learning has become a common analytical approach for modeling the relationship between measures of biological systems and clinical outcomes. Models generated from machine learning can be used for prediction, in which case the biological basis for the pattern being modeled may not be of interest. However, biological interpretation of machine learning models can be extremely important if the goal is to generate biological hypotheses that need to be validated clinically or experimentally. As such, methods for model interpretation have become an important component of machine learning research. A group of methods provide a graphical description of the model's global behavior i.e. partial dependency plots [1] and decision tree surrogate models. Several methods such as individual conditional expectation (ICE) plots [2], local interpretable model-agnostic explanations or LIME [3] and shapley additive explanations or SHAP and [4] focus on explaining individual model predictions. Another class of methods focus on assigning weights to individual variables or features based on how much information they provide to the predictions being made by the machine learning model. This latter approach generates 'feature importance scores' that can be used to create a list of features ranked according to their importance. This allows the modeler to focus on the most important features for biological interpretation. Interpretability (along with performance) is the key quality of 
the machine learning model, specifically when it is applied to biomedical research goals such as biomarkers discovery and patient diagnostics.

It has been widely discussed that for the complex biomedical phenotypes epistatic interactions between genes could be present more frequently than we commonly think [5-7]. Indeed, gene-gene interactions have been detected in multiple genome-wide association studies of various disease phenotypes, including Alzheimer's disease [8], cataracts [9], diabetes [10,11], cardiovascular diseases [12, 13], neurological diseases $[14,15]$, and various cancer types $[16,17]$. Epistasis has been defined in several different ways $[18,19]$. Here, we employ epistasis term as interactions between two or more gene loci such that the phenotype cannot be accurately predicted by simply adding the effects of individual gene loci. This is a statistical definition of epistasis as it measures the deviation from additivity using models that summarize genotypic and phenotypic variability of human population data. In contrast to that, biological epistasis is identified at the cellular level in an individual as a result of a physical interactions among molecules within the biological network. The relationship between two epistatic concepts is a complicated matter in such that statistical epistasis doesn't literally translate into the biological [18].

The intrinsic complexity of the epistatic interactions creates several analytical and practical challenges for its detection via traditional statistical methods due to its inability to detect non-additive effects in the large volume of data from GWAS studies. Machine learning methods have more flexibility in its power to detect underlying complexity of genetic architecture and, therefore, has been widely used in epistasis discovery [20]. A large body of research has been accumulated on epistatic interaction detection with neural network, support vector machines, multifactor dimensionality reduction and random forest (RF) models [21, 22]. Specifically, RF algorithm is known for its ability to take into account non-additive effects through its hierarchical tree-based structure [20,23]. A number of studies have been conducted on the integration of RF method into the epistatic interaction discovery. Among them RF with sliding window sequential forward feature selection method [24], a hybrid of RF and mutual information network methodology that reports reduction in RF bias towards the marginal effects [25], synthetic feature RF complemented with statistical epistasis network approach that incorporates genetic interactions within and among the biochemical pathways to explain its association with the disease outcome [26], mixed RF approach that accounts for both population structure and epistatic interactions [27], relative recurrency variable importance metric (r2VIM) [28] for RF that generates variable set with main and interaction effect, and permuted RF method that identifies interacting pair of SNPs by calculating the effect of disrupted interaction on the RF prediction error rate [29].

In addition to notorious performance on datasets with epistasis, benchmark studies have recognized the RF classification algorithm to be among the best classifiers for the majority of the real-world datasets [30, 31]. $\mathrm{RF}$ is conveniently interpretable with built-in feature importance scores implemented in a majority of popular programming languages and analytics platforms including Python and R. These are calculated with entropy or Gini importance criterion. Despite the utility of RF feature important scores, some studies have reported a bias introduced by the RF feature importance scores when working with categorical, grouped, and varying types of features [32]. An alternative way to estimate feature importances with the RF classifier is by calculating permutation feature importance (PFI) scores. This metric employs an exhaustive permutation concept where features are permuted one at a time and the importance scores expressed via the difference in Loading [MathJax]/jax/output/CommonHTML/jax.js 
the $\mathrm{ML}$ algorithm's performance score. While computationally intensive for large feature sets, this approach is advantageous due to its applicability to any machine learning method of any complexity and, therefore, is independent of the characteristics of any given algorithm.

In this study, we aim to improve the interpretability of RF predictions for genetic data in the presence of nonadditive interactions by comparing two feature importance metrics: RF's built-in feature importance coefficients (BIC) and PFI coefficients. We use two real-world datasets with previously described non-additive interactions to compare model interpretation using the two different feature importance score metrics. We also compare the metrics using simulated data with varying levels of interaction, imbalance in case/control ratio, and sample size where the ground truth is known.

\section{Results}

\section{Evaluation of feature importance metrics performance with HIBACHI simulated datasets.}

An RF classification algorithm with Gini impurity criterion was fitted on all simulated replicates with epistatic interactions. For majority of them, predictive balanced accuracy with 10 -fold cross-validation was estimated as 1.0 or very close to 1.0 (Fig. S1). PFI and BIC metrics were estimated for the fitted RF models and further compared to the real feature importances retrieved with the HIBACHI sensitivity analysis. The percentage of successful rank identification per 100 replicates of each experiment was reported in Table 1. For all combinations of factors that were considered in HIBACHI simulations, PFI metric consistently outperformed $\mathrm{BIC}$ metric in the ability to determine feature importance order. The most accurate PFI evaluation was produced for the datasets in the category with sample size 1000 , two-way IG and $50 \%$ of cases, where the most important feature (F1) was identified precisely in $90 \%$ of replicates, $\mathrm{F} 2-89 \%$, and $\mathrm{F} 3-77 \%$. For the sample size 10000 category, the most accurate estimate by PFI was done for the three-way IG, with $25 \%$ of cases where F1 was identified correctly for $88 \%$ of replicates, F2 - for $79 \%$, F3 -for $88 \%$, F4 - for $83 \%$. Overall, feature ranks estimated by PFI metric were at least twice more accurate than BIC estimates for nearly all the corresponding experiments. 
Table 1

$\mathrm{PFI}$ and BIC success in identification of feature ranks in datasets with two-way and three-way epistatic interactions. It is expressed as the percentage of a match of a metric rank's estimate with the true feature rank that was retrieved with the HIBACHI sensitivity analysis.

\section{Sample size 1000}

\section{Two-Way IG}

\begin{tabular}{lllllllll}
\hline Percent of cases: & $\mathbf{2 5 \%}$ & & $\mathbf{5 0 \%}$ & \multicolumn{2}{c}{$\mathbf{2 5 \%}$} & \multicolumn{3}{c}{$\mathbf{5 0 \%}$} \\
\hline Metrics: & PFI & BIC & PFI & BIC & PFI & BIC & PFI & BIC \\
\hline F1 & $70 \%$ & $40 \%$ & $90 \%$ & $42 \%$ & $79 \%$ & $38 \%$ & $78 \%$ & $18 \%$ \\
\hline F2 & $63 \%$ & $40 \%$ & $89 \%$ & $42 \%$ & $68 \%$ & $33 \%$ & $60 \%$ & $35 \%$ \\
\hline F3 & $93 \%$ & $83 \%$ & $77 \%$ & $81 \%$ & $78 \%$ & $33 \%$ & $71 \%$ & $18 \%$ \\
\hline F4 & $16 \%$ & $16 \%$ & $15 \%$ & $17 \%$ & $73 \%$ & $54 \%$ & $11 \%$ & $14 \%$ \\
\hline F5 & $5 \%$ & $4 \%$ & $4 \%$ & $5 \%$ & $33 \%$ & $31 \%$ & $5 \%$ & $6 \%$
\end{tabular}

\section{Sample size 10000}

Two-Way IG

\section{Percent of cases:}

Metrics:

F1

F2

F3

F4

F5

$25 \%$

$\mathrm{PFI}$

$78 \%$

$78 \%$

$99 \%$

$13 \%$

$5 \%$

PFI BIC

$50 \%$

Three-Way IG

F1, F2, etc. - feature ranks, PFI-permutation feature importance, BIC - build-in coefficients, IG information gain.

Interestingly, for the majority of replicates of the experiments with the two-way IG, the PFI metric was able to identify the top three features, while for the three-way IG this came up to the top four features. We investigated further into the population of the feature importance effect sizes and discovered that experiments with the two-way interactions observe the same range of effect sizes for the top two important features, while the effect sizes of the bottom two features are 2-3 orders of magnitude smaller than the top ones or zero (Fig. 3A, B). To support this observation Table 2 report the percentage of replicates with zero effect size at each feature importance rank: for the majority of the experiments with the two-way IG, two out of five features didn't have an effect on the phenotype. A more thorough exploration of the fitness function landscape confirmed that in the majority of cases only one pair or trio of features had enough time to evolve strong epistatic interactions due to the limited simulation time. Hence, we observe that the interacting pair of Loading [MathJax]/jax/output/CommonHTML/jax.js ution towards the phenotype, while non-interacting features 
contribution effect is orders of magnitude smaller and insufficient to be detected accurately by both importance metrics (Fig. 4A, B).

Table 2

Percentage of the features with zero effect size for every rank position.

\begin{tabular}{|c|c|c|c|c|}
\hline \multicolumn{5}{|l|}{ Sample size 1000} \\
\hline Information gain: & IG2 & & IG3 & \\
\hline Percent of cases: & $25 \%$ & $50 \%$ & 25 & $50 \%$ \\
\hline F1 & $\nabla 0 \%$ & $\mathbb{\otimes 0 \%}$ & $\varangle 0 \%$ & $\varangle 0 \%$ \\
\hline F2 & $0 \%$ & $0 \%$ & $0 \%$ & $0 \%$ \\
\hline F3 & $0 \%$ & $15 \%$ & $0 \%$ & $0 \%$ \\
\hline F4 & $76 \%$ & $70 \%$ & $23 \%$ & $83 \%$ \\
\hline F5 & $91 \%$ & $92 \%$ & $65 \%$ & $92 \%$ \\
\hline \multicolumn{5}{|c|}{ Sample size 10000} \\
\hline Information gain: & IG2 & & IG3 & \\
\hline Percent of cases: & $25 \%$ & $50 \%$ & $25 \%$ & $50 \%$ \\
\hline F1 & $\bowtie 0 \%$ & $\mathbb{\nabla 0 \%}$ & $\mathbb{\otimes 0} \%$ & $\varangle 0 \%$ \\
\hline F2 & $0 \%$ & $0 \%$ & $0 \%$ & $0 \%$ \\
\hline F3 & $0 \%$ & $17 \%$ & $0 \%$ & $0 \%$ \\
\hline F4 & $85 \%$ & $54 \%$ & $16 \%$ & $47 \%$ \\
\hline F5 & $94 \%$ & $84 \%$ & $59 \%$ & $88 \%$ \\
\hline
\end{tabular}

F1, F2, etc. - feature ranks, PFI-permutation feature importance, BIC - build-in coefficients, IG information gain.

Evaluation of the feature importances in real-world datasets with epistatic interactions.

We used HIBACHI simulation framework to obtain datasets with strong epistatic interaction between the features - genetic variants and the phenotype. We were able to demonstrate that regardless of the dataset's parameters, the best method to determine features' informative contribution to the phenotype is PFI. To validate our findings for real-world data we analyzed two datasets with complex disease phenotype which previously have been identified to have epistatic interactions among SNPs. The subset of seven SNPs was selected from the Alzheimer's disease data and the subset of six SNPs was selected from the Glaucoma dataset as described above (see Methods 2.4). A ViSEN analysis and plot for the Alzheimer's dataset (Fig. 5A) revealed one SNP with large independent effect affiliated with ApoE gene (rs429358, MI 0.08) - a known risk fantarfar Alshnimardinanon and thron atrang two-way gene-gene interactions (rs4955208 - rs7782571, IG Loading [MathJax]/jax/output/CommonHTML/jax.js 
0.05; rs12785149 - rs12209418, IG 0.05; rs2414325 - rs1931073, IG 0.05). ViSEN analysis of the Glaucoma dataset revealed two strong gene-gene interactions (rs7738052 - rs1489169, IG 0.015, and rs10915315 - or rs1266924, IG 0.015). We built a predictive model for each dataset with the RF classifier: the model was tuned with grid search and had classification balanced accuracy $62.6 \%$ for the Alzheimer dataset, and $60 \%$ for the Glaucoma dataset. We verified the significance of the cross-validated balanced accuracy scores of the optimized classifiers by doing a permutation test [33] and confirmed that both models have reliable classification performance (Fig. 6). We further calculated PFI and BIC estimates for the optimized RF models. For Alzheimer dataset (Fig. 7A) the most important feature, according to both PFI and BIC metrics, is the SNP with the largest independent effect - rs429358. This SNP has been discovered by ViSEN analysis and expected to have strong effect on the phenotype. However, the consecutive feature importance order diverged between two metrics. According to PFI rank, SNPs located at second and third position belong to the same interaction and valued with the highest IG out of all calculated pairwise interactions for this dataset (Fig. 5A). At the same time, SNPs located at the second and third position by BIC ranking didn't create a strong pairwise interactions with each other. We observed a similar discrepancy between the metric rank evaluations for the Glaucoma datasets (Fig. 7B): while SNP rs2157719 has the largest main effect among all SNPs and was assigned to be the top feature by both metrics, the following order of SNPs was outlined conversely by different metrics. SNP rs10915315 has the largest number of detected pairwise interaction among all SNPs and is ranked second by PFI, while only fourth by BIC; SNP rs1489169 has three pairwise interactions detected and is ranked third by PFI and second by BIC. Interestingly, both metrics identified the less important and non-important SNPs in the same order, suggesting that the issue with the discrepancy between the metrics evaluations should be address for the top features only.

\section{Discussion}

Multiple research studies suggested that epistatic interactions are widespread by nature and that many genes work in an interactive manner [5]. Epistatic interactions are expected to be found in a variety of pathophysiological processes with one of them most likely to be Alzheimer's disease [8]. The most powerful predictor of Alzheimer's disease at this time is ApoE E4 gene variation: one or two copies of ApoE is associated with an increased risk of disease onset [34]. However, some carriers of ApoE E4 variation haven't developed an Alzheimer's disease so it is very likely that other genetic factors are involved in disease's pathophysiology. ViSEN entropy-based analysis revealed several strong pairwise genetic interactions, along with the known largest independent signal from the ApoE variant (rs429358) (Fig. 5A). Furthermore, ViSEN method allocated epistatic interactions within the Glaucoma disease dataset: several strong pairwise interactions in addition to the independent main effect contribution from the SNP affiliated with retinal ganglion cells pathology (rs2157719) have been confirmed (Fig. 5B). Evaluation of an informative contribution of a single genetic factor involved in the two- or three-way interactions towards the phenotypic outcome is a challenging analytical task and it requires a non-linear solution which RF classifier is notorious for. Therefore, we aimed to identify whether RF classifier is capable to detect genetic signatures that were previously confirmed by ViSEN analysis and whether different RF's feature importance metrics will be able to agree on the rank. 
Two feature importance metrics were considered, PFI and BIC, and each was compared after RF analysis of data derived from genome-wide association studies of Glaucoma and Alzheimer's. The resulting feature ranking confirms the lack of consensus between the studied metrics (Fig. 6). Indeed, while BIC and PFI identified the SNPs with the largest independent main effects (rs429358 and rs2157719) as a top feature in both real-world datasets, genes involved in the epistatic interactions have been assigned different importance ranks by different metrics. More specifically, in the Alzheimer data, the second and the third most important feature predicted by PFI belong to the same interacting pair (rs4955208 and rs7782571), while the same rank positions predicted by BIC were occupied by SNPs that do not belong to the same genetic interaction (Fig. 5A, Fig. 7A). A similar discrepancy has been observed in the Glaucoma dataset: PFI and BIC indicated different interacting pairs of SNPs as the second and third most important feature (Fig. 7A), however SNP predicted to be the second most important by PFI is a part of four additional SNP-SNP interactions, while SNP predicted second by BIC is a part of only three interacting SNP pairs (Fig. 5B).

Such uncertainty has been associated with RF predictions in the past and we attempted to reveal the true interpretation with the computational experiments driven by HIBACHI simulations. The HIBACHI framework has the ability to consider any desirable biological concept in the form of mathematical expressions that define the genotype-phenotype relationship and evolve models that can be used to simulate data consistent with that relationship. We set up a simulation goal to maximize two- or three-way interactions among features and compared RF's feature importance metrics with the sensitivity analysis results of the simulated data that provided us with the ground truth information about the feature ranks (Fig. 2). In all HIBACHI experimental setups, which included such factors as the proportion of cases and controls, sample size and interaction complexity, PFI metrics produced the most precise feature ranking (Table 1, Fig. 3). Although BIC metric misplaced feature ranks for the majority of the replicates, it correctly identified features that belong to the interactive pair or trio by putting them as a top two or three features correspondingly. Therefore, we can suggest that BIC metric can still be used with some caution, however, when there is a need for an absolute precision, PFI estimation method should be used.

PFI has some limitations we didn't cover in our experiments - it is particularly sensitive to highly correlated features. In case such features are present in the dataset, PFI requires a special preprocessing directed onto the removal of such features. An example of this could be a hierarchical clustering based on the Spearman's rank correlation coefficients with following cluster-based filtering. In future studies, a more advanced permutation feature importance techniques may be considered which can include pairwise permutation importance, joint importance by maximal subtrees, and joint variable importance as well as corrected Gini importance score [35]. In addition, a more complex epistatic schemes needs to be explored (e.g. different combinations of marginal and interaction effects as in [36], multiple strong interactions and/or combination of two- and three-way interactions). Within our simulation framework we mostly observed one or two strong interactions and this might be an overly simplistic given the complexity of common diseases such as Alzheimer's and glaucoma.

\section{Conclusion}


In this study, we performed a comparative analysis of feature importance metrics with the aim to improve Random Forest's interpretability in datasets with complex interactions. By analyzing both real and simulated data, we established that the permutation feature importance metric provides more precise feature importance rank estimation in the presence of non-additive interactions.

\section{Methods}

\section{Random forest and its properties}

$\mathrm{RF}$ is a popular $\mathrm{ML}$ algorithm because it often demonstrates good performance while remaining relatively easy to optimize and interpret. RF algorithms belong to a Bagging (Bootstrap Aggregation) type of ensemble ML methods where a group of weak learners in a form of decision trees (DT) classify the outcome using majority vote. Decision trees are sensitive to the data they are trained and often suffer from high variance problem especially when the depth of the tree is high. To address that RF algorithm trains each tree on a subset of samples drawn from the complete dataset with the bootstrap procedure. An additional source of randomness within the RF algorithm is introduced during the construction of a tree when selecting a split node from the random sample of features (in place of the greedy search through all feature set like in DT). These two sources of randomness aim to decorrelate weak learners and correspondingly decrease the variance of an estimator by combining diverse trees prediction via majority vote.

During the construction of a DT, the decrease in the error function can be calculated for a feature at each split node. In a classification task, this is often done via estimating the Gini score or the entropy score. The function decreases can be averaged across all trees and returned as feature importances score (the greater the decrease the higher feature importance). Feature importance scores are often conveniently implemented as a RF function which makes this method more interpretable.

\section{Permutation Feature Importances}

Permutation feature importance metrics were first introduced by Breiman in his Random Forest manuscript [37] and further extended by Altmann [38] to correct for the bias of the RF's Gini importance and entropy criterion for feature selection. We utilize a custom implementation of PFI which could be applied to any machine learning classification and regression algorithms (Fig. 1). Here, PFI metric is calculated with following steps: 1 ) the dataset is shuffled and split into the training and testing datasets 2) the model is fitted on the training dataset and the balanced accuracy is estimated on the testing dataset, 3) feature 1 out of $\mathrm{N}$ is permuted for the testing dataset 4) the balanced accuracy is estimated on the permuted testing dataset 5) the relative decrease of the permuted and non-permuted balanced accuracies is calculated and stored as relative decrease in accuracy, 4) step 2 and 3 are repeated for the remaining $\mathrm{N}-1$ features, 5) steps 1 through 4 are replicated for $\mathrm{N}-1$ times with the new seeds for shuffle and split procedure, 6) mean of relative decrease in accuracy per feature is calculated across the splits and is used as features' PFI value. Retrieved PFI values were normalized to sum to 1 . 


\section{Evaluation Of The Feature Importance Metrics \\ Data simulation using HIBACHI}

We used Heuristic Identification of Biological Architectures for simulating Complex Hierarchical Interactions (HIBACHI) software to simulate genetic datasets with non-additive epistatic interactions of different complexity. The HIBACHI method employs biological and mathematical frameworks to connect genotype and phenotype $[39,40]$ At the bottom of the biological framework is the concept of information transfer from the DNA sequence to a clinical phenotype through complex interactions at multiple levels: gene expression, pathway, and cell. Within this framework, a population of samples is expressed as a collection of genes (genotype) each of which has three variants 0,1 or 2 . The mathematical framework's goal is to specify a relationship between genotype and phenotype in terms of logical, arithmetical and other functions. HIBACHI merges the frameworks by evolving a mathematical expression tree which when applied to a genotype, generates a binary clinical phenotype.

HIBACHI employs Genetic Programming (GP) as an optimization engine. One of the key characteristics of GP is a fitness function which is represented through the mathematical expression that satisfy a specific objective of interest defined by user. This objective could be a performance of a machine learning pipeline, complexity of genetic interactions, odds ratio of genetic effect sizes, length of expression tree, etc. or a combination of those in the form of the multi-objective fitness function. Additionally, user to allowed to specify the length of the optimization (by specifying the number of generations), the population size (number of samples) and genotype size (number of genes) of the dataset.

At the beginning of the GP optimization process, a population of individuals with random mathematical expression trees is initialized and is further subjected to mutational and recombinational processes. This process serves as a source of variation for the expression trees and have a pre-defined rate. After that, a userdefined fitness function is calculated and the best-fitted individuals are selected for the next round. At the last optimization round, an overall best-fitted individual is used as an output dataset.

For the aims established within this study, we wanted to generate datasets with non-additive epistatic interactions that would involve two and three genetic variants and, therefore, we have defined a fitness function that maximizes two-way and three-way information gain (IG) term. Entropy-based IG approach to detect epistatic interactions has been introduced by Moore et al. [41] and extended by Fan et al. [42]. Two-way IG defined as:

$$
I G(X ; Y ; Z)=I(X, Y ; Z)-I(X ; Z)-I(Y ; Z)
$$

Where / is mutual information that describes the dependency between variables $\mathrm{X}, \mathrm{Y}$ and $\mathrm{Z}$. It measures the reduction of uncertainty of one variable $(Z)$ given the knowledge of others $(X$ and $Y$ ) It is expressed with entropy terms: 
Where entropy $H$ defined with the probability mass function:

$$
\begin{gathered}
H(Z \mid X)=H(Z, X)-H(Z) ; H(Z)=-\sum_{Z \in Z} p(z) \log p(Z) \\
H(Z, X)=-\sum_{Z \in Z} \sum_{X \in X} p(Z, X) \log p(Z, X)
\end{gathered}
$$

Definitions of three-way IG can be found in Hu et al., 2013 [43]. We used the following version of this term: $I G(X ; Y ; Z ; N)=I(X, Y, Z ; N)-I G(X ; Y ; N)-I G(X ; Z ; N)-I G(Y ; Z ; N)-I(X ; N)-I(Y ; N)-I(Z ; N)$

Additionally, a second fitness objective was set up as maximization of expression tree length, to encourage multiple combinations of genetic interactions. In addition to the variability in the interaction complexity, the following factors have been considered in the HIBACHI experimental schemes: percent of cases (25\% and $50 \%$ ) to address an imbalanced dataset structure, and sample size (1000 and 10000). Each experimental setup was reproduced 100 times using random seed generator and the whole population of replicates was considered in the consecutive analysis. All simulated data used here is available upon request.

\section{Sensitivity Analysis}

We implemented a HIBACHI-based sensitivity analysis to determine the true feature importances ranks and the effect sizes. For this the permutation-based framework was implemented with the following steps (Fig. 2): 1) split HIBACHI-generated dataset into the outcome vector and the feature set 2) permute feature vector 1 out of $\mathrm{N}$ and re-evaluate the outcome by applying the HIBACHI-generated expression tree 3) calculate the dissimilarity of the outcome as of mismatch between the perturbed and unperturbed feature set 4) repeat the estimate 2) for the remaining feature and normalize the counts by the total sum; 5) replicate steps 2-4 100 times and calculate the average of the replicates per feature as a final true feature importance score. Sensitivity scores were further normalized to sum to 1.

\section{Real world data analysis.}

To examine the convergence of the RF's feature importance metrics we used two real-world datasets with evidence for non-additive interactions. The first includes preselected SNPs from a genome-wide association study of Alzheimer's Disease while the second includes preselected SNPs from a genome-wide association study of Primary Open Angle Glaucoma (POAG). The Alzheimer's dataset came from the Alzheimer's Disease Neuroimaging Initiative during which functional MRI was taken every six to twelve months for patients with three health conditions (neuro-typical (identified here as controls), and mild cognitive impairment and Alzheimer's disease (identified here as cases)). A computational evolution system [44] identified a model of seven SNPs with evidence of non-additive interactions and a classification accuracy of 0.738 . Among these SNPs are the SNP with the large main effect that is located in the APOE gene (rs429358) - a known risk factor for Alzheimer disease, four SNPs located within genes with known functionality/disease state (rs1931073 - an intergenic region near the PPAP2B gene that is participating in cell-cell interactions, m770n571_nnarthn Ienn nannthat in nampciated with the Walker-Walburg syndrome, rs4955208 - in the 
OSBPL10 genes which are expressed into intracellular lipid receptor, rs12209418 - in the PKIB gene that codes a protein kinase inhibitor) and two remaining SNPs (rs2414325, rs12785149) are located within genes with unknown functionality.

The glaucoma dataset came from the Glaucoma Gene Environment Initiative study and contained with POAG individuals identified as cases and healthy individuals as controls. This dataset has been previously analyzed by Moore at al. [45] with the EMERGENT algorithm that resulted in the identification of a model of six SNP's with evidence of non-additive interactions and a classification accuracy of 0.615. Two of these SNPs (rs2157719, and rs1266924) are located within the genes that were previously associated with glaucoma disease, two SNPs (rs10915315, and rs1489169) are located within the genes that are associated with glaucoma-non-related diseases and relevant pathology, and two more SNPs (rs936498, and rs7738052) are located within the genes that were not previously associated with any disease, but have a known functionality that is relevant to visual cortex and retina development.

We used the visualization of the statistical interaction network (ViSEN) method [46] to analyze and visualize SNP main effects, and two-way and three-way gene-gene interactions among SNPs for real-world datasets. The ViSEN method calculates the mutual information (MI) between individual SNP (genotype) and the phenotype, the pairwise interaction between every pair of SNPs and the phenotype and the three-way interaction between every combination of three SNPs and the phenotype via the IG term. A positive IG indicates synergistic (i.e. non-additive) effects of SNPS on the phenotype. The IG metrics used in the ViSEN method were designed to detect pure epistatic interactions and excluded all lower-order effects (by subtracting all main effects and pairwise synergies in cases of the three-way term).

\section{Abbreviations}

RF- Randon Forest, PFI

permutation feature importance, HIBACHI - Heuristic Identification of Biological Architectures for simulating Complex Hierarchical Interactions, BIC-build-in coefficients

\section{Declarations}

\section{Ethics approval and consent to participate}

Human data used in preparation of this article were obtained from the Alzheimer's Disease Neuroimaging Initiative (ADNI) database (adni.loni.usc.edu) and The Primary Open-Angle Glaucoma Genes and Environment (GLAUGEN) Study at dbGaP database.

\section{Consent for publication}

Not applicable 
The authors declare that they have no competing interests

\section{Funding}

This work was supported by National Institutes of Health (USA) grants LM010098 and Al116794

\section{Authors' contributions}

AO conceived the study, performed data simulation and data analysis. JHM conceived and supervised the study. All authors were involved in writing the manuscript. All authors read and approved the manuscript.

\section{Acknowledgments}

Not applicable

\section{References}

1. Hastie T, Tibshirani R, Friedman J, The Elements of Statistical Learning, Second Edition, Sect. 10.13.2, Springer, 2009.

2. Goldstein A, et al. "Peeking inside the black box: Visualizing statistical learning with plots of individual conditional expectation." Journal of Computational and Graphical Statistics 24.1 2015; 44-65.

3. Ribeiro MT, Singh S, Guestrin C "Why should I trust you?": Explaining the predictions of any classifier. In International Conference on Knowledge Discovery and Data Mining (KDD), 2016.

4. Lundberg SM, Lee SI. A unified approach to interpreting model predictions. Proc. Adv. Neural Inf. Process. Syst. 2017; 4768-4777.

5. Moore JH. The ubiquitous nature of epistasis in determining susceptibility to common human diseases. Hum Hered. 2003;56:73-82.

6. Carlborg O, Haley CS, Epistasis. Too often neglected in complex trait studies? Nat Rev Genet. 2004;5:618-25. doi:10.1038/nrg1407.

7. Mackay TF, Moore JH. Why epistasis is important for tackling complex human disease genetics. Genome Med. 2014;6:42.

8. Hohman TJ, Bush WS, Jiang L, et al. Discovery of gene-gene interactions across multiple independent data sets of late onset Alzheimer disease from the Alzheimer Disease Genetics Consortium. Neurobiol Aging. 2016;38:141-50. doi:10.1016/j.neurobiolaging.2015.10.031.

9. Pendergrass SA, Verma SS, Holzinger ER, Moore CB, Wallace J, Dudek SM, Huggins W, Kitchner T, Waudby C, Berg R, McCarty CA, Ritchie MD. Next-generation analysis of cataracts: determining knowledge driven gene-gene interactions using Biofilter, and gene-environment interactions using the PhenX Toolkit. Pac Symp Biocomput. 2013;147 - 58. Corrected and republished in: Pac Symp Biocomput. 2015;:495-505. PMID: 23424120; PMCID: PMC3615413. 
10. Bell JT, Timpson NJ, Rayner NW, Zeggini E, Frayling TM, et al. Genome-wide association scan allowing for epistasis in type 2 diabetes. Ann Hum Genet. 2011;75:10-9.

11. Manduchi E, Chesi A, Hall MA, Grant SFA, Moore JH. Leveraging putative enhancer-promoter interactions to investigate two-way epistasis in Type 2 Diabetes GWAS. Pac Symp Biocomput. 2018;2018:548-58.

12. $10.1038 /$ srep01099

Lippert C, Listgarten J, Davidson RI, et al. An exhaustive epistatic SNP association analysis on expanded Wellcome Trust data [published correction appears in Sci Rep. 2013 Feb 18;3:1321. Poong H. Sci Rep. 2013;3:1099. doi:10.1038/srep01099.

13. Meng Y, Groth S, Quinn JR, Bisognano J, Wu TT. 2017An exploration of gene-gene interactions and their effects on hypertension. Int. J. Genomics. 2017, 7208318.

14. Sha Q, Zhang Z, Schymick JC, Traynor BJ, Zhang S. Genome-Wide Association Reveals Three SNPs Associated With Sporadic Amyotrophic Lateral Sclerosis Through a Two-Locus Analysis. BMC Med Genet. 2009;10:86. 86.

15. Steffens M, Becker T, Sander T, Fimmers R, Herold C, Holler D, Leu A, Herms C, Cichon S, Bohn S, Gerstner B, Griebel T, Nöthen M, Wienker MM, Baur TF. M, P: Feasible and Successful: Genome-Wide Interaction Analysis Involving All 1.9 × $10^{11}$ Pair-Wise Interaction Tests. Hum Hered. 2010;69:268-84. doi:10.1159/000295896.

16. Chu M, Zhang R, Zhao Y, Wu C, Guo H, Zhou B, Lu J, Shi Y, Dai J, Jin G, Ma H, Dong J, Wei Y, Wang C, Gong J, Sun C, Zhu M, Qiu Y, Wu T, Hu Z, Lin D, Shen H, Chen F. A genome-wide gene-gene interaction analysis identifies an epistatic gene pair for lung cancer susceptibility in Han Chinese. Carcinogenesis. 2014 Mar;35(3):572-7. doi:10.1093/carcin/bgt400. Epub 2013 Dec 9. PMID: 24325914; PMCID: PMC3941747.

17. Shen Z, Li Z, Song J, Chen Y, Shi. Genome-wide two-locus interaction analysis identifies multiple epistatic SNP pairs that confer risk of prostate cancer: a cross-population study. Int J Cancer. 2017;140(9):207584. 10.1002/ijc.30622.

18. Moore JH, Williams SM. Traversing the conceptual divide between biological and statistical epistasis: systems biology and a more modern synthesis. Bioessays. 2005;27:637-46. doi:10.1002/bies.20236.

19. Phillips P. Epistasis - the essential role of gene interactions in the structure and evolution of genetic systems. Nat Rev Genet. 2008;9:855-67. https://doi.org/10.1038/nrg2452.

20. McKinney BA, Reif DM, Ritchie MD, Moore JH. Machine learning for detecting gene-gene interactions: a review. Appl Bioinformatics. 2006;5(2):77-88. doi:10.2165/00822942-200605020-00002.

21. Moore JH, Asselbergs FW, Williams SM. Bioinformatics challenges for genome-wide association studies. Bioinformatics. 2010;26(4):445-55. doi:10.1093/bioinformatics/btp713.

22. $10.1155 / 2013 / 432375$

Koo CL, Liew MJ, Mohamad MS, Salleh AH. (2013). A Review for detecting gene-gene interactions using machine learning methods in genetic epidemiology. Biomed. Res. Int. 2013:432375. doi: 10.1155/2013/432375.

23. Goldstein BA, Polley EC, Briggs FBS. Random forests for genetic association studies. Stat Appl Genet Mnl Rinl $2011 \cdot 1 \cap(1) \cdot 22$ 
24. Jiang R, Tang W, Wu X, Fu W. A random forest approach to the detection of epistatic interactions in casecontrol studies. BMC Bioinformatics. 2009.

25. Pan QX, Hu T, Malley JD, Andrew AS, Karagas MR, Moore JH, “Supervising random forest using attribute interaction networks," in Evolutionary Computation, Machine Learning and Data Mining in

Bioinformatics, L. Vanneschi, W. S. Bush, and M. Giacobini, Eds., vol. 7833 of Lecture Notes in Computer Science. 2013;104-116.

26. Pan Q, Hu T, Malley JD, Andrew AS, Karagas MR, Moore JH. A System-Level Pathway-Phenotype Association Analysis Using Synthetic Feature Random Forest. Genet Epidemiol. 2014;38:209-19. doi:10.1002/gepi.21794.

27. Stephan J, Stegle O, Beyer A. A random forest approach to capture genetic effects in the presence of population structure. Nat Communications 6, 7432 (2015). https://doi-

org.proxy.library.upenn.edu/10.1038/ncomms8432.

28. Holzinger EM, Szymczk S, Dasgupta A, Malley J, Li Q, Bailey-Wilson JE. (Jan 4-8, 2015) Variable Selection Method for the Identification of Epistatic Models. Paper presented at the Pacific Symposium on Biocomputing (PSB), Maui, HI.

29. Li J, Malley JD, Andrew AS. Kargas MR. Moore JH. Detecting gene-gene interactions using a permutationbased random forest method. BioDataMining2016; 9, 14 https://doi.org/10.1186/s13040-016-0093-5.

30. Fernandez-Delgado M, Cernadas E, Barro S, Amorim D. Do we need hundreds of classifiers to solve real world classification problems? J Mach Learn Res. 2014;15:3133-81.

31. PMLB: a large benchmark suite for machine learning evaluation and comparison https://doi.org/10.1186/s13040-017-0154-4

Olson RS, La Cava W, Orzechowski P, et al. PMLB: a large benchmark suite for machine learning evaluation and comparison. BioData Mining. 2017; 10, 36. https://doi.org/10.1186/s13040-017-0154-4.

32. Strobl C, Boulesteix AL, Zeileis A, Hothorn T. Bias in random forest variable importance measures: illustrations, sources and a solution. BMC Bioinformatics. 2007;8:25. doi:10.1186/1471-2105-8-25. Published 2007 Jan 25.

33. Ojala and Garriga. Permutation Tests for Studying Classifier Performance. The Journal of Machine Learning Research (2010) vol. 1.

34. Corder EH, et al. Gene dose of apolipoprotein E type 4 allele and the risk of Alzheimer's disease in late onset families. Science. 1993;261:921-3.

35. 10.1093/bioinformatics/bty373

Nembrini S, König IR, Wright MN, The revival of the Gini importance?, Bioinformatics, Volume 34, Issue 21, 01 November 2018, Pages 3711-3718, https://doi.org/10.1093/bioinformatics/bty373.

36. Wright MN, Ziegler A, König IR. Do little interactions get lost in dark random forests? BMC Bioinformatics. 2016;17:145. doi:10.1186/s12859-016-0995-8.

37. Breiman L, Random F. Mach Learn. 2001;45:5-32.

38. Altmann A, Toloşi L, Sander O, Lengauer T. Permutation importance: a corrected feature importance measure. Bioinformatics. 2010;26(10):1340-7. 
39. Moore JH, Amos R, Kiralis J, Andrews PC. Heuristic identification of biological architectures for simulating complex hierarchical genetic interactions. Genet Epidemiol. 2015;39(1):25-34.

doi:10.1002/gepi.21865.

40. Moore JH, Shestov M, Schmitt P, Olson RS. A heuristic method for simulating open-data of arbitrary complexity that can be used to compare and evaluate machine learning methods. Pac Symp Biocomput. 2018;23:259-67.

41. Moore JH, Gilbert JC, Tsai CT, Chiang FT, Holden T, Barney N, White BC. 2006 A flexible computational framework for detecting, characterizing, and interpreting statistical patterns of epistasis in genetic studies of human disease susceptibility. J Theor Biol 241: 252-261.

42. Fan $\mathrm{R}$, Zhong $\mathrm{M}$, Wang $\mathrm{S}$, et al. Entropy-based information gain approaches to detect and to characterize gene-gene and gene-environment interactions/correlations of complex diseases. Genet Epidemiol. 2011;35:706-21.

43. Hu T, Chen Y, Kiralis JW, Collins RL, Wejse C, Sirugo G, Williams SM, Moore JH. An information-gain approach to detecting three-way epistatic interactions in genetic association studies. J Am Med Inform Assoc. 2013.

44. Moore JH, Douglas P, Saykin, Andrew S, Li. 2013. Exploring Interestingness in a Computational Evolution System for the Genome-Wide Genetic Analysis of Alzheimer's Disease. Third Indonesian-American Kavli Frontiers of Science Symposium. Bali, Indonesia.

45. Moore JH, Greene CS, Hill DP. Identification of Novel Genetic Models of Glaucoma Using the "EMERGENT" Genetic Programming-Based Artificial Intelligence System. In: Riolo R, Worzel WP, Kotanchek M, editors. Genet. Program. Theory Pract. XII [Internet]. Springer International Publishing; 2015 [cited 2016 Dec 12]. p. 17-35.

46. Hu T, Chen Y, Kiralis JW, et al. ViSEN: methodology and software for visualization of statistical epistasis networks. Genet Epidemiol. 2013;37:283-5.

\section{Figures}


Replicate 100 times with the different split seed
1. Shuffle and split the dataset

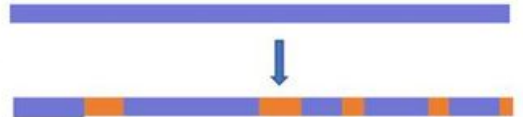

2. Fit and predict the performance accuracy $A$

3. Permute feature $x$

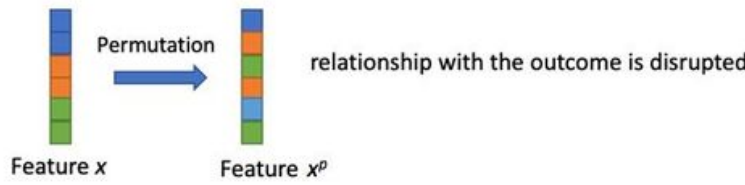

4. Predict the performance accuracy on the permuted testing set $A_{x}^{p}$

5. Calculate relative decrease in accuracy for feature $x: \Delta A_{x}=\frac{A-A_{x}^{p}}{A}$

6. Calculate mean decrease in accuracy for each of $\mathrm{N}$ feature as $\mathrm{PFI} \quad P F I_{x}=\frac{\sum_{i}^{100} \Delta A_{x i}}{100}$

Replicate for $\mathrm{N}$ features

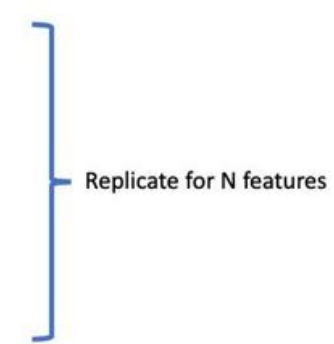

\section{Figure 1}

Scheme of the permutation feature importance custom implementation

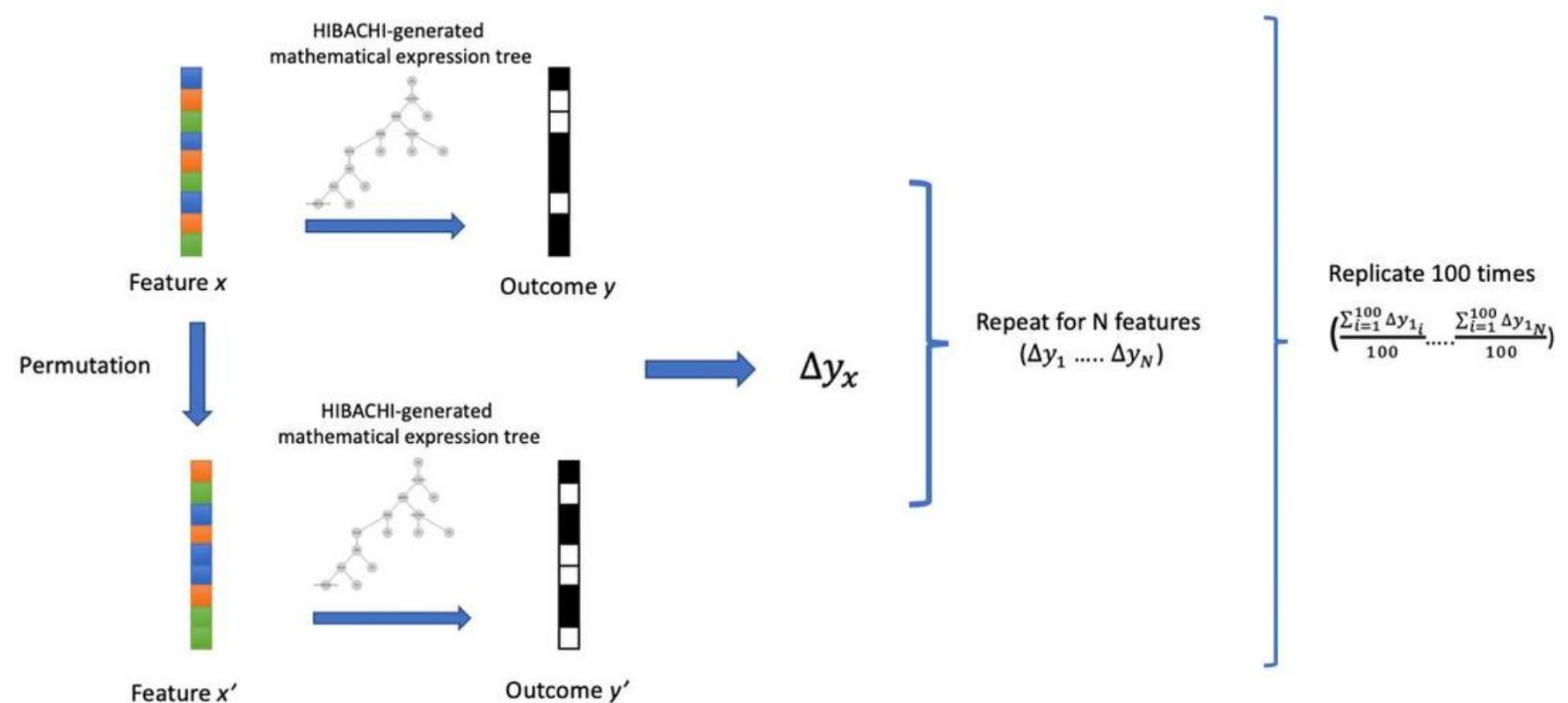

\section{Figure 2}

Scheme of sensitivity analysis for the HIBACHI experiments

Loading [MathJax]/jax/output/CommonHTML/jax.js 

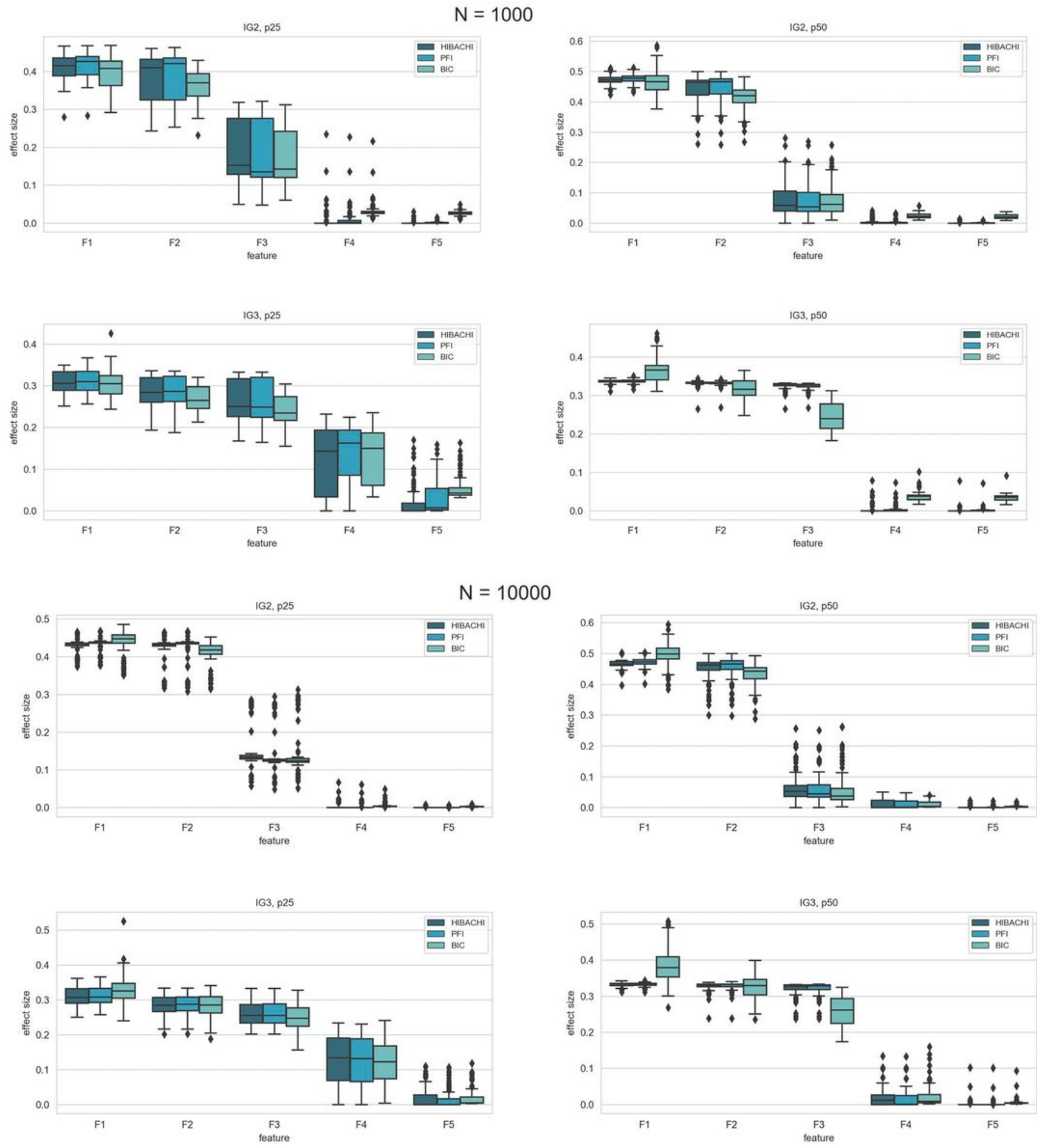

Figure 3

Effect size per feature rank estimated by PFI, BIC and HIBACHI sensitivity analysis for sample size 1000 (A) and 10000 (B). F1, F2, etc. - feature ranks, PFI -permutation feature importance, BIC - build-in coefficients, IG- Information Gain, p25, p50 - percentage of cases 

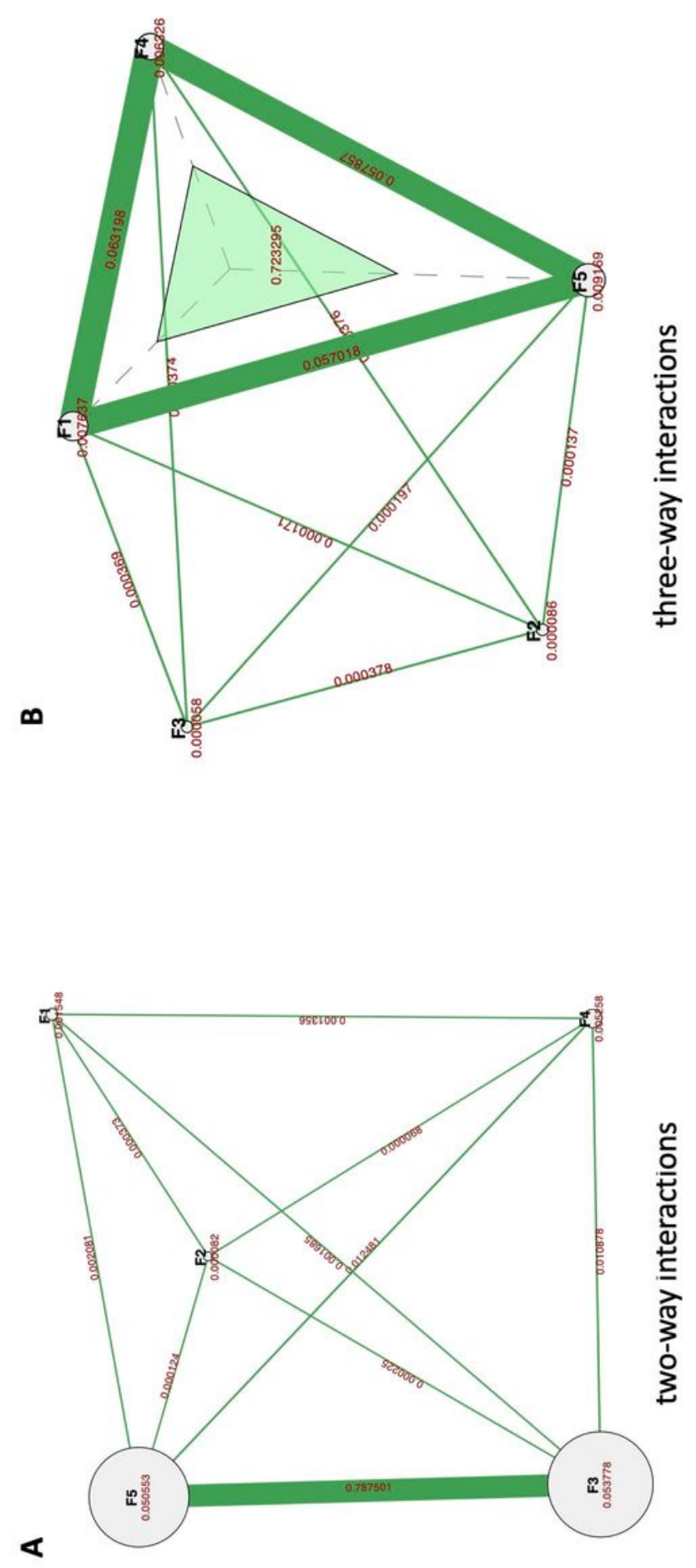

Figure 4

Example of ViSEN plots for the datasets with two-way (A) and three-way (B) interactions. Features main effects, two-way and three-way IG values are noted respectively. 

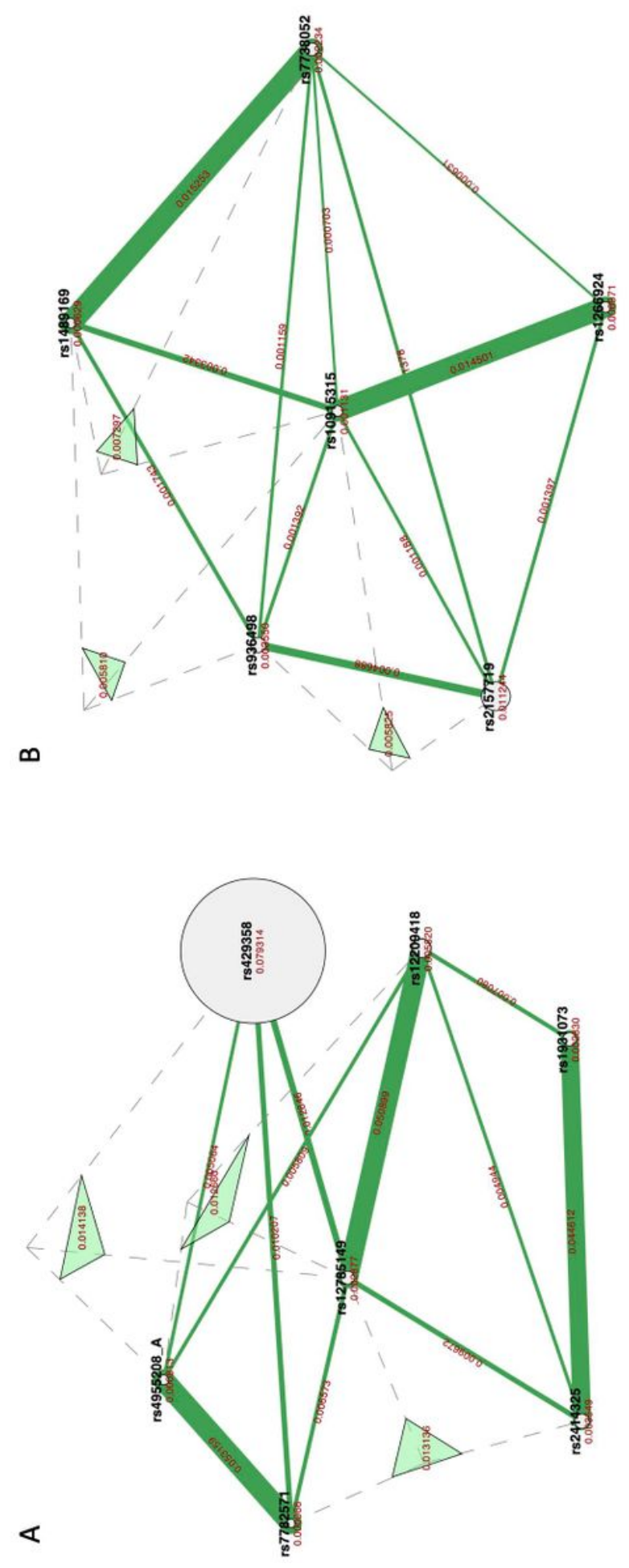

Figure 5

ViSEN plots for selected SNPs in Alzheimer's (A) and Glaucoma (B) datasets. SNPs main effects, two-way and three-way IG values are noted respectively. 
A

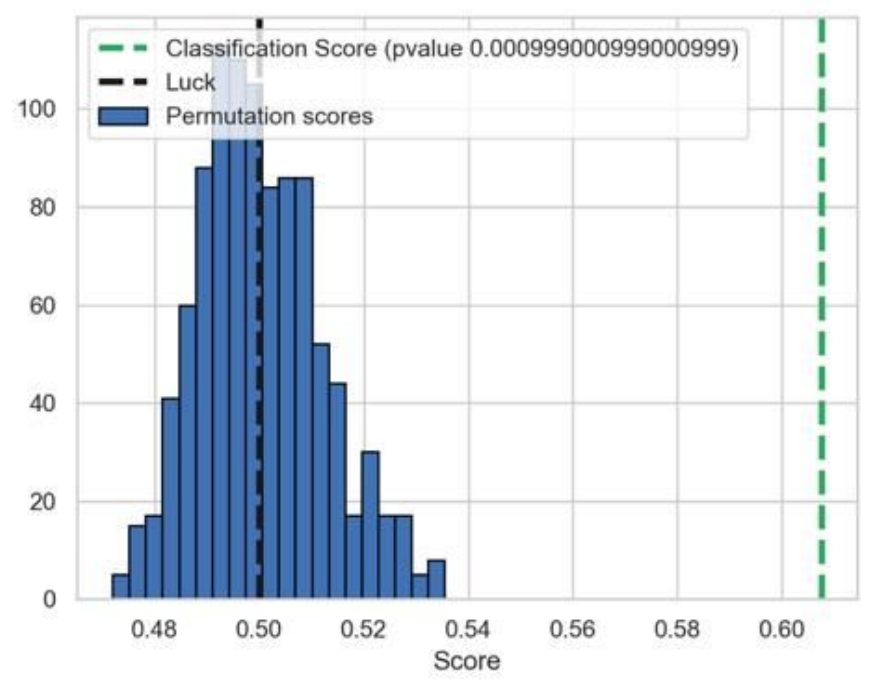

\section{B}

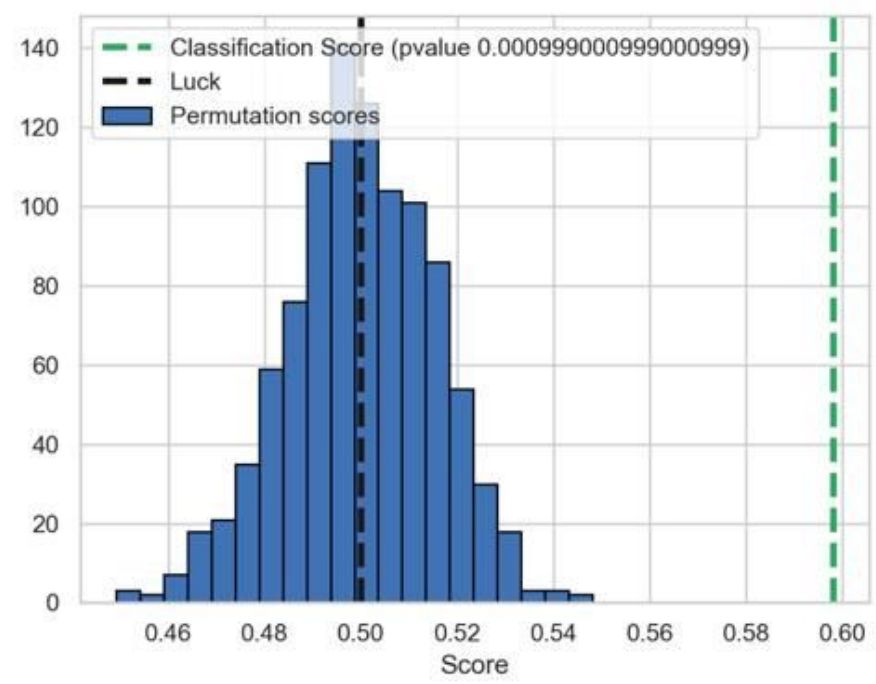

\section{Figure 6}

Permutation test scores for the RF classifier for Alzheimer's (A) and Glaucoma (B) datasets. 


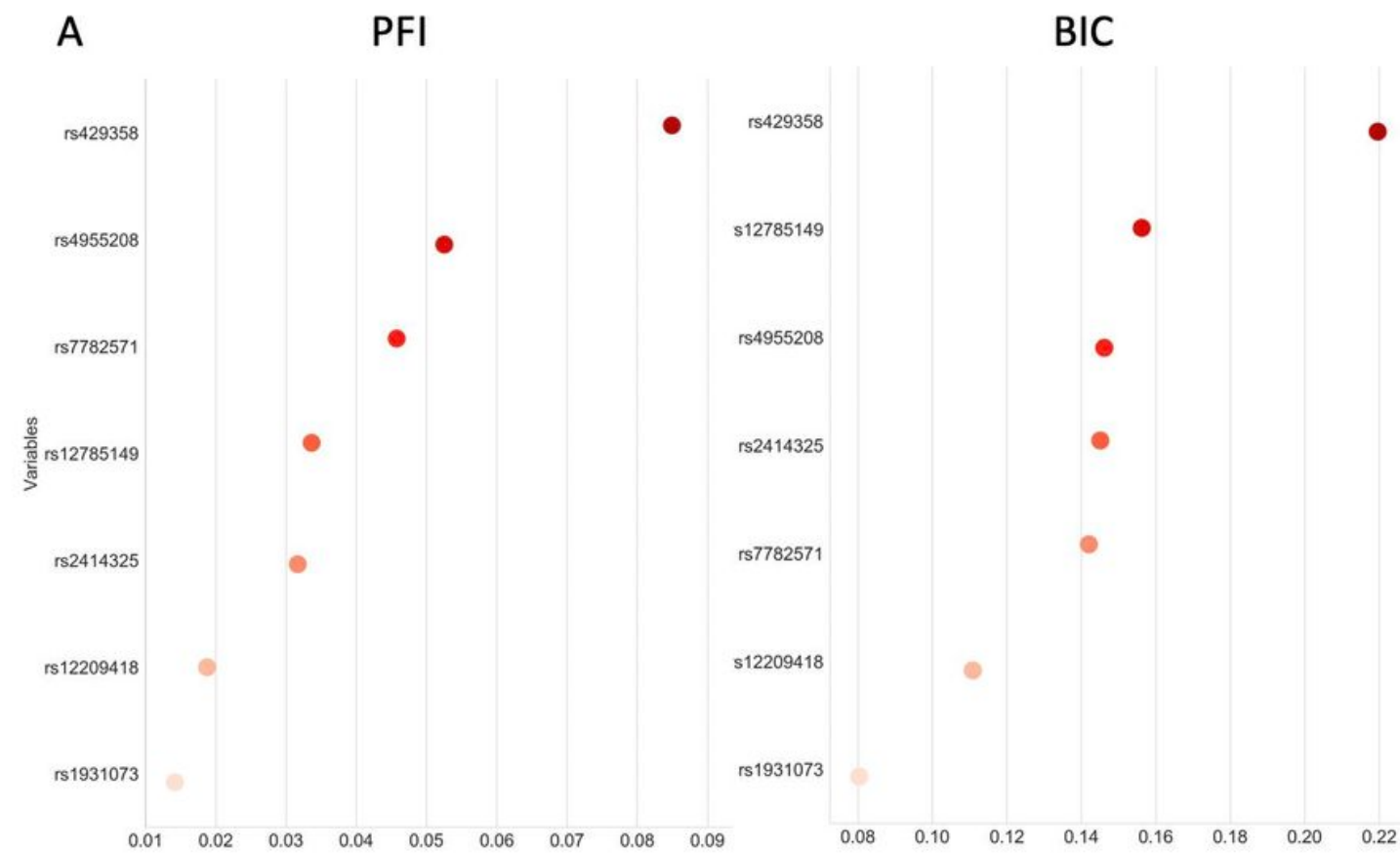

B

$\mathrm{PFI} \quad \mathrm{BIC}$

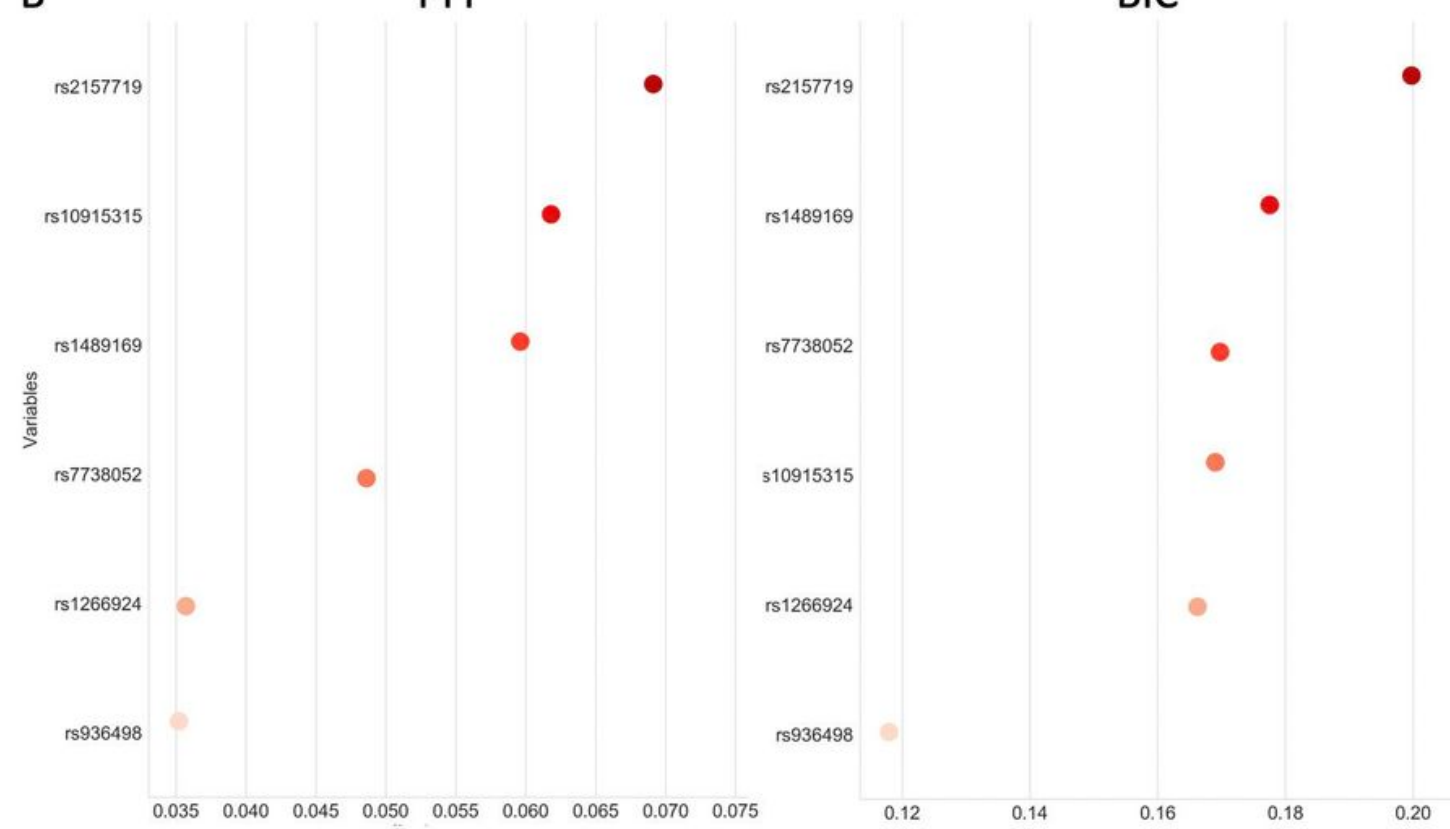

Figure 7

PFI and BIC estimates for Alzheimer's (A) and Glaucoma (B) datasets. PFI -permutation feature importance, BIC - build-in coefficients

\section{Supplementary Files}

This is a list of sunnlementarv files asconiated with this preprint. Click to download. Loading [MathJax]/jax/output/CommonHTML/jax.js 
- Figures1.docx

Loading [MathJax]/jax/output/CommonHTML/jax.js

Page 23/23 